\title{
ANALISI DELL'EFFICIENZA ENERGETICA DELLA BIBLIOTECA COMUNALE DI CAMPO MOURÃO-PR: PARAMETRI DEL PROGRAMMA NAZIONALE DI ETICHETTATURA (RTQ-C)
}

\section{ARTICOLO ORIGINALE}

CARVALHO, Fanny Islana de Lima ${ }^{1}$, HALMEMAN, Maria Cristina Rodrigues ${ }^{2}$, LERCO, Felipe Matos dos Santos ${ }^{3}$

CARVALHO, Fanny Islana de Lima. HALMEMAN, Maria Cristina Rodrigues. LERCO, Felipe Matos dos Santos. Analisi dell'efficienza energetica della biblioteca comunale di Campo Mourão-PR: parametri del programma nazionale di etichettatura (RTQ-C). Revista Científica Multidisciplinar Núcleo do Conhecimento. Anno. 06, Ed. 10, Vol. 05, pp. 43-62. Ottobre 2021. ISSN: 2448-0959, Link di accesso:

https://www.nucleodoconhecimento.com.br/ingegneria-ambientaleit/biblioteca-comunale, DOI: 10.32749/nucleodoconhecimento.com.br/ingegneriaambientale-it/biblioteca-comunale

\section{RIEPILOGO}

Il settore delle costruzioni, responsabile degli impatti ambientali come la produzione di rifiuti, l'acqua e il consumo di energia, ha come sfida attuale quella di portare l'efficienza energetica nel cantiere e nell'ambiente post-costruito. Pertanto, in relazione al consumo di energia, INMETRO ha sviluppato un programma di etichettatura volto a classificare l'efficienza energetica delle apparecchiature elettriche e anche degli edifici. Si scopre che gli edifici pubblici sono tenuti a ottenere l'etichetta nazionale di conservazione dell'energia (ENCE). Alla ricerca di quanto sopra, si chiede come misurare l'efficienza energetica degli edifici pubblici,

\footnotetext{
${ }^{1}$ Laurea in Architettura e Urbanistica. ORCID: 0000-0002-1871-2618.

${ }^{2}$ Laurea in Ingegneria Civile. ORCID: 0000-0002-2809-6601.

${ }^{3}$ Dottorato di Ricerca in Scienze Agronomiche. ORCID: 0000-0001-6964-7572.
}

RC: 100247

Disponibile in: https://www.nucleodoconhecimento.com.br/ingegneria-ambientaleit/biblioteca-comunale 
rendendoli energeticamente appropriati, e quindi soddisfare le esigenze dell'utente, fornendo comfort ambientale ed economia. L'obiettivo di questo lavoro era simulare I'ottenimento dell'etichetta ENCE Geral e presentare il livello di efficienza energetica della Biblioteca Comunale di Campo Mourão-PR. È stato utilizzato il Metodo Prescrittivo, stabilito dal Regolamento Tecnico di Qualità per il Livello di Efficienza Energetica degli Edifici Commerciali, di Servizio e Pubblici (RTQ-C). Sono stati analizzati tre sistemi costruttivi: Involucro, Sistema di illuminazione e Sistema di aria condizionata. Di conseguenza, l'edificio in studio ha presentato una classificazione di livello $C$ di efficienza, su una scala $A$ - $E$, essendo $A$ molto efficiente ed $E$ inefficiente. $\mathrm{Si}$ conclude che l'edificio non offre comfort agli utenti, oltre all'elevato consumo energetico. Tuttavia, è possibile adottare misure che possono aiutare a ottimizzare il livello di efficienza energetica in loco.

Parole chiave: Involucro, Sistema di illuminazione, Aria condizionata, Metodo prescrittivo, Classificazione totale.

\section{INTRODUZIONE}

La preoccupazione dei paesi sviluppati per la conservazione ambientale ha portato a conferenze internazionali, alla ricerca di alternative economiche. (DEBATES INTERDISCIPLINARES VII, 2016). Esempi di queste conferenze sono il Protocollo di Kyoto e la Convenzione di Stoccolma. Nel panorama nazionale, dal 2014 in poi, gli edifici pubblici sono tenuti a presentare un'etichetta di efficienza energetica (ENCE), come pubblicato sulla Gazzetta Ufficiale dell'Unione dal Dipartimento di Logistica e Tecnologia della norma IN02/2014 (CONSELHO DE ARQUITETURA E URBANISMO/BR, 2014). Premesso quanto sopra, è sorta la domanda su come sia possibile misurare l'efficienza energetica di un edificio pubblico, garantendo comfort ed economia agli utenti.

II Programma di etichettatura brasiliano (PBE), insieme all'EBE Edifica, è nato dalle discussioni avviate dall'Istituto Nazionale di Metrologia, Qualità e Tecnologia

RC: 100247

Disponibile in: https://www.nucleodoconhecimento.com.br/ingegneria-ambientaleit/biblioteca-comunale 
(INMETRO). L'obiettivo di questo programma è quello di sensibilizzare i consumatori attraverso l'informazione sul funzionamento delle apparecchiature e sul loro consumo energetico (CONFERÊNCIA DE ESTUDOS EM ENERGIA ELÉTRICA, 2019). Questo programma ha sviluppato il regolamento tecnico di qualità per il livello di efficienza energetica degli edifici residenziali (RTQ-R) e i requisiti tecnici di qualità per il livello di efficienza energetica degli edifici commerciali, di servizio e pubblici (RTQ-C). II sistema di etichettatura misura il livello di efficienza energetica su una scala "A - E", essendo "A" più efficiente e "E" meno efficiente. RTQ-C valuta gli edifici attraverso due metodi: metodo di simulazione (eseguito dal software) e metodo prescrittivo (realizzato attraverso equazioni).

Esistono quattro tipi di etichette, che sono: ENCE Generale (tiene conto di tre sistemi di costruzione: involucro, illuminazione e aria condizionata); ENCE Parziale (valuta autonomamente ogni sistema); ENCE Simulazione (ottenuta in fase di progetto); e ENCE Edificio costruito (può essere richiesto dopo aver ottenuto il permesso di lavoro o comprovati allacciamenti di energia elettrica e gas da parte dei concessionari).

Questo articolo mirava ad individuare il livello di Efficienza Energetica della Biblioteca Comunale simulando l'ottenimento di ENCE generale. L'analisi è stata sviluppata in conformità con il manuale applicativo RTQ-C versione 4 del 2017. Per raggiungere I'obiettivo generale, questo lavoro è stato sviluppato sulla base dei seguenti obiettivi specifici: Osservare i prerequisiti richiesti dal Manuale per l'applicazione di RTQ-C; raccogliere dati sul sito ed elaborare informazioni di progettazione architettonica; calcolare e determinare il livello di efficienza energetica per i tre sistemi dell'edificio.

RC: 100247

Disponibile in: https://www.nucleodoconhecimento.com.br/ingegneria-ambientaleit/biblioteca-comunale 


\section{MATERIALI E METODI}

\subsection{MATERIALI}

L'attuale Biblioteca Comunale Insegnante Egydio Martello si trova nella zona centrale di Campo Mourão-PR. La città ha circa 96.102 abitanti (IBGE, 2020). Campo Mourão è un hub di COMCAM (Comunità dei Comuni della Regione campo Mourão), che rappresenta venticinque comuni, in questo modo, la città offre supporto per la sua microregione attraverso cure mediche, istruzione, industria e altre aree.

Figura 01. Ubicazione della Biblioteca Comunale.

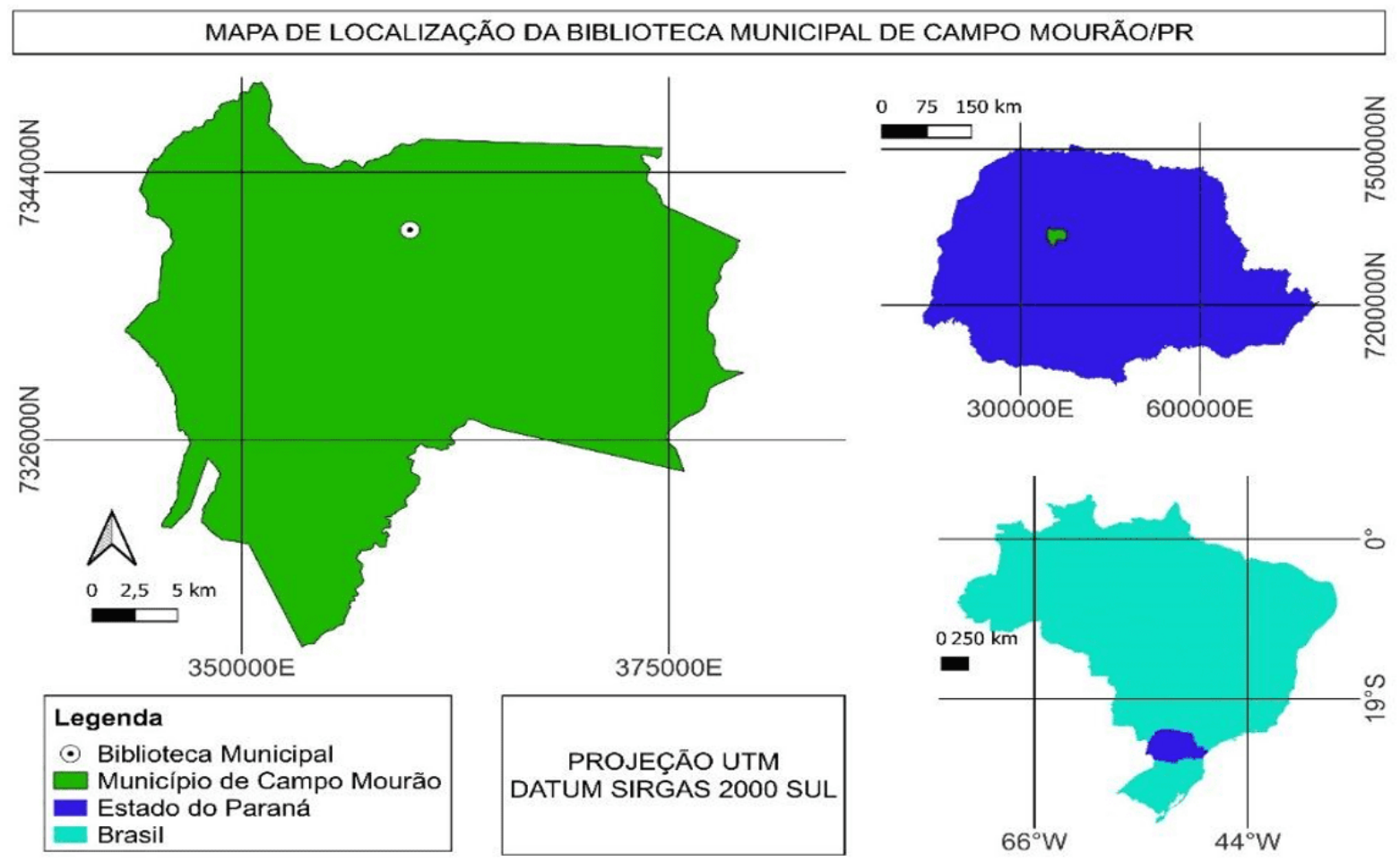

Fonte: Jairo Jerônimo de Campos Filho (2021).

L'edificio in studio ha una grande rilevanza storica nel contesto dello sviluppo del comune, poiché è stato originariamente progettato per essere il terminal degli

RC: 100247

Disponibile in: https://www.nucleodoconhecimento.com.br/ingegneria-ambientaleit/biblioteca-comunale 
autobus. Successivamente, l'edificio è stato adattato per ospitare la biblioteca, che ha aperto il 30 dicembre 2004.

Figura 02. Vecchia stazione degli autobus di Campo Mourão (A), attuale Biblioteca Comunale (B), Biblioteca Municipale della Facciata Principale (C).



Fonte: (A) Collezione personale di Jair Elias dos Santos Júnior (1969) e $(B, C)$ collezione della Biblioteca Comunale Professor Egydio Martello (2007).

Oltre a una vasta collezione, uno spazio espositivo artistico, una ludoteca e aree di studio, la biblioteca ospita anche l'Accademia di Lettere Mourãoense. Si tratta di un edificio lineare, a due piani, in struttura in cemento armato, con una superficie totale costruita di 1772,92 $\mathrm{m}^{2}$, secondo il layout della Biblioteca in Figura 03. Gran parte del suo involucro è costituito da tende di vetro traslucido, che favoriscono il riscaldamento indesiderato degli spazi interni. $\dot{E}$ importante notare che la sua

RC: 100247

Disponibile in: https://www.nucleodoconhecimento.com.br/ingegneria-ambientaleit/biblioteca-comunale 
facciata principale è rivolta verso nord-est, quindi riceve un'alta incidenza di radiazione solare nel periodo estivo.

Figura 03. Layout Biblioteca Piano Terra (A), Uffici Piano Terra (B), Piano Superiore Biblioteca (C).

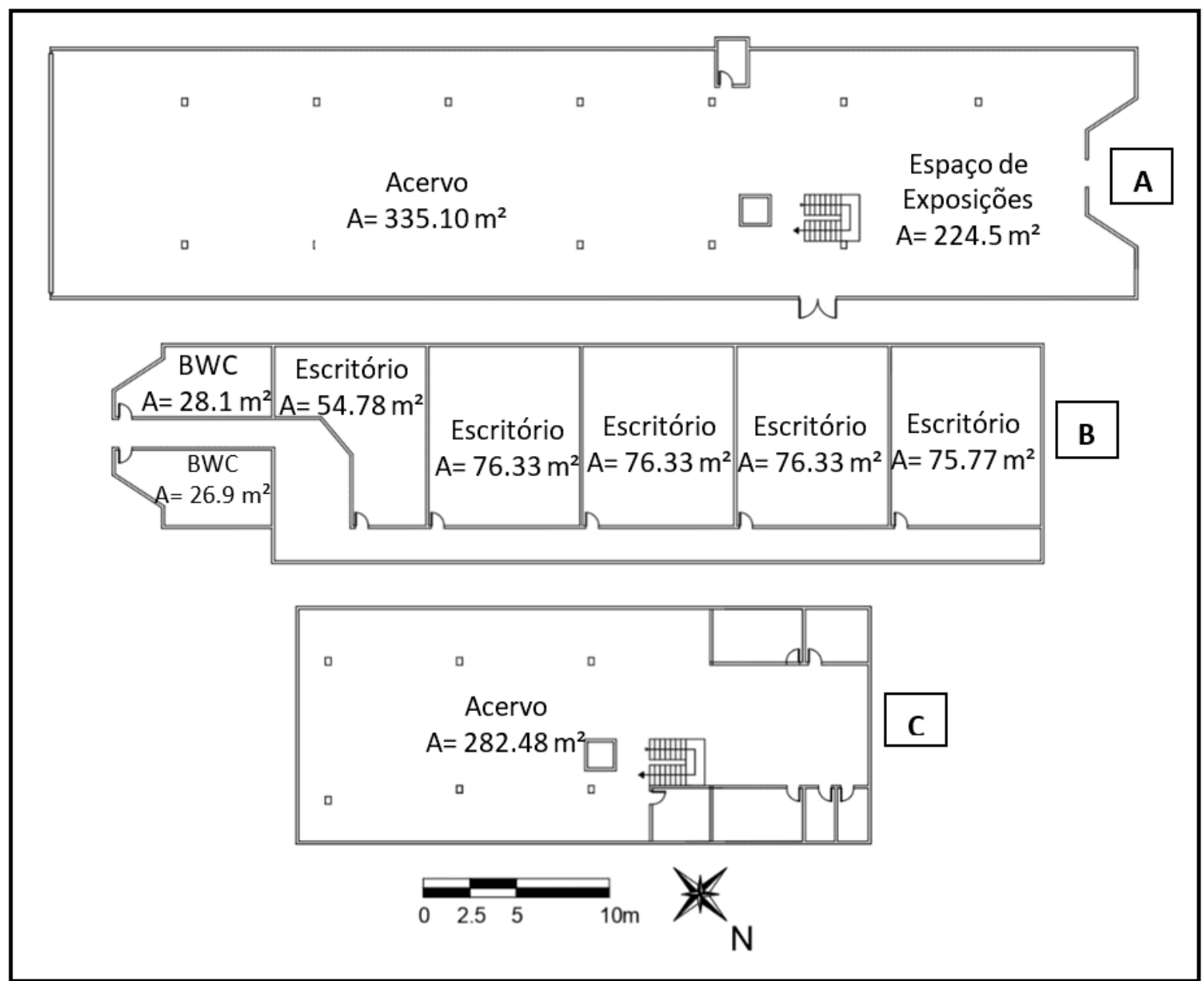

Fonte: Adattato dal progetto architettonico originale (2003).

\subsection{METODI}

II metodo adottato per il presente studio è il metodo prescrittivo, che si applica solo agli edifici condizionati e valuta l'efficienza energetica attraverso equazioni, secondo la Zona Bioclimatica e la superficie totale dell'edificio. Per ottenere punteggi per

RC: 100247

Disponibile in: https://www.nucleodoconhecimento.com.br/ingegneria-ambientaleit/biblioteca-comunale 
l'etichetta generale ENCE, sono stati analizzati tre sistemi: Involucro, Sistema di illuminazione e Aria condizionata. Questa metodologia richiede la valutazione dei prerequisiti generali e specifici del sistema, che saranno discussi di seguito.

\subsubsection{PREREQUISITI GENERALI}

Per i livelli A, B e C, ci sono due prerequisiti generali. II mancato rispetto di essi influisce sul punteggio finale dell'edificio.

- L'edificio deve avere un circuito elettrico distinto per ogni uso, che si tratti di apparecchiature di condizionamento d'aria, lampade o altri. Tuttavia, il Manuale per l'applicazione di RTQ-C (2017) stabilisce che questa voce non è obbligatoria per gli edifici costruiti prima del 2009, pertanto l'edificio in fase di studio ne è esente.

- Riscaldamento dell'acqua: questo prerequisito si applica solo negli edifici commerciali o di servizio, dove il consumo di acqua calda è superiore al 10\% della domanda totale di energia. Nel caso dell'oggetto di studio, non vi è alcuna installazione di scaldabagni, pertanto questa voce non si applica.

\subsubsection{EQUAZIONI PER LA PROCEDURA DI CALCOLO}

Nel processo di classificazione individuale dei sistemi, così come per la classificazione generale dell'efficienza, sono state utilizzate equazioni secondo la Tabella 01.

RC: 100247

Disponibile in: https://www.nucleodoconhecimento.com.br/ingegneria-ambientaleit/biblioteca-comunale 
Tabella 01. Tabella generale delle equazioni.

\begin{tabular}{|c|c|c|}
\hline NOME & EQUAÇÃO & VARIÁVEIS \\
\hline $\begin{array}{c}\text { Equação } \\
01\end{array}$ & $\begin{array}{c}\text { IC env }=-14,14 . F A- \\
113,94 . F F+50,82 . P A F t o t a l+4,86 . F S- \\
\text { 0,32.AVS }+0,26 \cdot A H S-\frac{35,75}{F F}- \\
\text { 0,54.PAFtotal.AHS }+277,98\end{array}$ & $\begin{array}{l}\text { IC env: Indicador de Consumo da } \\
\text { Envoltória. } \\
\text { Ape: Área de projeção do edifício } \\
\left(\mathrm{m}^{2}\right) \text {; } \\
\text { Ato: Área total construída }\left(\mathrm{m}^{2}\right) ; \\
\text { Aenv: Área da envoltória }\left(\mathrm{m}^{2}\right) ; \\
\text { Apcob: Área de projeção da } \\
\text { cobertura }\left(\mathrm{m}^{2}\right) ; \\
\text { AVS: Ângulo Vertical de } \\
\text { Sombreamento } \\
\text { AHS: Ângulo Horizontal de } \\
\text { Sombreamento } \\
\text { FF: Fator de Forma, (Aenv/ Vtot); } \\
\text { FA: Fator Altura, (Apcob/ Atot); } \\
\text { FS: Fator Solar; } \\
\text { PAF } \mathrm{T} \text { : Percentual de Abertura na } \\
\text { Fachada total: média do percentual } \\
\text { de aberturas existentes; }\end{array}$ \\
\hline
\end{tabular}

RC: 100247

Disponibile in: https://www.nucleodoconhecimento.com.br/ingegneria-ambientaleit/biblioteca-comunale 


\begin{tabular}{|c|c|c|}
\hline $\begin{array}{c}\text { Equação } \\
05\end{array}$ & $\begin{array}{l}\mathbf{P T}=0,3 \cdot\left\{\left(\text { EqNumEnv } \frac{A C}{A U}\right)+\right. \\
\left.\left(\frac{A P T}{A U} \cdot 5+\frac{A N C}{A U} \cdot \text { EqNu } \mathrm{mV}\right)\right\}+0,3 . \\
(\text { EqNumDPl })+0,4 \cdot\{(\text { EqNumCA } . \\
\left.\left.\frac{A C}{A U}\right)+\left(\frac{A P T}{A U} \cdot 5+\frac{A N C}{A U} \cdot \text { EqNumV }\right)\right\}+ \\
\mathrm{b}_{0}^{1}\end{array}$ & $\begin{array}{l}\text { EqNumEnv: Equivalente Numérico } \\
\text { Envoltória } \\
\text { AC: Área Condicionada } \\
\text { AU: Área útil } \\
\text { APT: Área de permanência } \\
\text { Transitória } \\
\text { ANC: Área não condicionada } \\
\text { EqNumV: Equivalente Numérico } \\
\text { Área Ventilada } \\
\text { EqNumDPI: Equivalente Numérico } \\
\text { Densidade de Potência Instalada } \\
\text { EqNumCa: Equivalente Numérico } \\
\text { Condicionamento de Ar } \\
\text { b: Bonificações }\end{array}$ \\
\hline & & $\begin{array}{l}\text { Vto }: \text { Volume total da edificação } \\
\left(\mathrm{m}^{3}\right) \text {; }\end{array}$ \\
\hline $\begin{array}{c}\text { Equação } \\
02\end{array}$ & $\mathbf{i}=\frac{I C \text { máx }-I C \text { mín }}{4}$ & $\begin{array}{l}\text { i: Intervalo } \\
\text { IC } \mathbf{C}_{\max } \text { Indicador de Consumo } \\
\text { máximo } \\
\text { IC } \mathbf{C}_{\min }: \text { Indicador de Consumo } \\
\text { Mínimo }\end{array}$ \\
\hline $\begin{array}{c}\text { Equação } \\
03\end{array}$ & $\mathbf{P}_{\text {lim }}=\mathrm{A}^{2}$. DPIL & $\begin{array}{l}\text { PI }_{\text {im: Potência Limite }} \\
\text { A: Área iluminada } \\
\text { DPIL: Densidade de Potência } \\
\text { Instalada Limite }\end{array}$ \\
\hline $\begin{array}{c}\text { Equação } \\
04\end{array}$ & EqNumCA $=\sum_{n=1}^{x}:\left[\begin{array}{l}\text { EqNumCA } \\
n\end{array} \frac{\text { Capn }}{\text { Capt }}\right]$ & $\begin{array}{l}\text { EqNumCA: Equivalente Numérico } \\
\text { de Condicionamento de } \mathrm{Ar} \\
\text { Capn: Capacidade de cada } \\
\text { sistema } \\
\text { Capt: Capacidade total dos } \\
\text { sistemas } \\
\text { x: Quantidade de sistemas }\end{array}$ \\
\hline
\end{tabular}

Fonte: Adattato dal Manuale per l'applicazione di RTQ-C (2017).

\subsubsection{INVOLUCRO}

Al fine di soddisfare i prerequisiti specifici del sistema dell'involucro, devono essere osservati i valori delle variabili descritte nel grafico 02.

RC: 100247

Disponibile in: https://www.nucleodoconhecimento.com.br/ingegneria-ambientaleit/biblioteca-comunale 
Tabella 02. Requisiti specifici per Involucro - Zona 3.

\begin{tabular}{|c|c|c|c|c|c|c|}
\hline Nível & \multicolumn{3}{|c|}{ Transmitância Térmica (U) } & \multicolumn{2}{|c|}{$\begin{array}{c}\text { Cores e } \\
\text { Absortância } \\
\text { Térmica (a) }\end{array}$} & $\begin{array}{c}\text { Iluminação } \\
\text { Zenital }\end{array}$ \\
\hline \multirow{2}{*}{ - } & \multicolumn{2}{|c|}{ Cobertura } & Parede & Cobertura & Parede & \multirow{7}{*}{ N/A } \\
\hline & $\begin{array}{c}\text { Ambiente } \\
\text { Condicionado }\end{array}$ & $\begin{array}{l}\text { Ambiente não } \\
\text { condicionado }\end{array}$ & - & - & & \\
\hline A & $1.0 \mathrm{~W} / \mathrm{m}^{2} \mathrm{~K}$ & $2.0 \mathrm{~W} / \mathrm{m}^{2} \mathrm{~K}$ & $\begin{array}{c}3.7 \\
W / m^{2} k\end{array}$ & $<0.50$ & $<0.50$ & \\
\hline B & $1.5 \mathrm{~W} / \mathrm{m}^{2} \mathrm{k}$ & $2.0 \mathrm{~W} / \mathrm{m}^{2} \mathrm{~K}$ & $\begin{array}{c}3.7 \\
\mathrm{~W} / \mathrm{m}^{2} \mathrm{k}\end{array}$ & $<0.50$ & $\mathrm{~N} / \mathrm{A}$ & \\
\hline C & \multirow{2}{*}{\multicolumn{2}{|c|}{$2.0 \mathrm{~W} / \mathrm{m}^{2} \mathrm{~K}$}} & 3.7 & \multirow{3}{*}{\multicolumn{2}{|c|}{$\mathrm{N} / \mathrm{A}$}} & \\
\hline D & & & $\mathrm{W} / \mathrm{m}^{2} \mathrm{k}$ & & & \\
\hline E & \multicolumn{2}{|c|}{$>2.0 \mathrm{~W} / \mathrm{m}^{2} \mathrm{~K}$} & $\begin{array}{c}>3.7 \\
\mathrm{~W} / \mathrm{m}^{2} \mathrm{~K}\end{array}$ & & & \\
\hline
\end{tabular}

Fonte: Adattato dal manuale di applicazione RTQ-C (2017).

ABNT NBR 15220-2 (2008) stabilisce i parametri per le prestazioni termiche degli edifici. Dopo aver verificato la frequenza dei valori di cui sopra in conformità con la norma, è necessario osservare la Zona Bioclimatica in cui è inserito l'oggetto di studio. Secondo l'Allegato 1 del Manuale per l'applicazione di RTQ-C (2017), la città di Campo Mourão si trova nella zona bioclimatica 3, come mostrato nella Figura 04.

RC: 100247

Disponibile in: https://www.nucleodoconhecimento.com.br/ingegneria-ambientaleit/biblioteca-comunale 
Figura 04. Zonizzazione bioclimatica del Brasile.



Fonte: Bioclimatismo (2021).

Per la procedura di calcolo sono state necessarie le informazioni estratte dal progetto architettonico e le variabili secondo la Tabella 01.

Considerando che il Manuale specifica diverse equazioni in base all'area dell'oggetto di studio, per le aree superiori a $500 \mathrm{~m}^{2}$ l'equazione $01 \mathrm{si}$ inserisce nell'edificio.

RC: 100247

Disponibile in: https://www.nucleodoconhecimento.com.br/ingegneria-ambientaleit/biblioteca-comunale 
Poco dopo, I'IC minimo e I'IC massimo sono stati determinati utilizzando la stessa equazione, ma con i valori di input secondo il grafico 03.

Tabella 03. Parametri IC massimi e IC minimi.

\begin{tabular}{|l|c|c|c|c|}
\hline & PAF & FS & AVS & AHS \\
\hline IC máximo & 0,60 & 0,61 & 0 & 0 \\
\hline IC mínimo & 0,05 & 0,87 & 0 & 0 \\
\hline
\end{tabular}

Fonte: Manuale per l'applicazione di RTQ-C (2017).

Pertanto, l'intervallo (i) di IC massimo e IC minimo è stato calcolato dall'equazione 02. Con il valore di (i), è stata definita la tabella 04 :

Tabella 04. Limiti degli intervalli dei livelli di efficienza.

\begin{tabular}{|c|c|c|c|c|c|}
\hline Eficiência & A & B & C & D & E \\
\hline Lim. Mín. & N/A & $\begin{array}{c}\text { IC máx }- \\
3 i+0,01\end{array}$ & $\begin{array}{c}\text { IC máx }- \\
2 i+0,01\end{array}$ & $\begin{array}{c}\text { IC máx }-\mathrm{i} \\
+0,01\end{array}$ & $\begin{array}{c}\text { IC máx }+ \\
0,01\end{array}$ \\
\hline Lim. Máx. & $\begin{array}{c}\text { IC máx }- \\
3 \mathrm{i}\end{array}$ & IC máx $-2 \mathrm{i}$ & $\mathrm{IC}$ máx $-\mathrm{i}$ & $\mathrm{IC}$ máx & $\mathrm{N} / \mathrm{A}$ \\
\hline
\end{tabular}

Fonte: Manuale per l'applicazione di RTQ-C (2017).

Infine, dai valori in Tabella 04, il valore di IC dell'involucro è stato confrontato con i limiti di IC massimo e IC minimo per identificare il livello di efficienza.

\subsubsection{SISTEMA DI ILLUMINAZIONE}

II rispetto dei prerequisiti specifici per il sistema di illuminazione è descritto nel grafico 05.

RC: 100247

Disponibile in: https://www.nucleodoconhecimento.com.br/ingegneria-ambientaleit/biblioteca-comunale 
Tabella 05. Prerequisiti per l'illuminazione.

\begin{tabular}{|l|l|l|l|l|}
\hline Pré-Requisito & Nível A & Nível B & Nível C & Situação \\
\hline 1. Divisão dos circuitos & Exigido & Exigido & Exigido & Isento \\
\hline 2. Contribuição da luz natural & Exigido & Exigido & N/A & $\begin{array}{l}\text { Não } \\
\text { atende }\end{array}$ \\
\hline $\begin{array}{l}\text { 3. Desligamento automático } \\
\text { do sistema de iluminação }\end{array}$ & Exigido & N/A & N/A & $\begin{array}{l}\text { Não } \\
\text { atende }\end{array}$ \\
\hline
\end{tabular}

Fonte: Adattato dal manuale di applicazione RTQ-C (2017).

L'edificio in studio è esente dal prerequisito numero 1 e non soddisfa gli altri prerequisiti, quindi il livello del sistema di illuminazione può automaticamente avere come classificazione massima solo il livello $\mathrm{C}$. Tuttavia, la sua classificazione finale è soggetta ai calcoli del limite di densità di potenza installata.

Esistono due metodi per calcolare l'efficienza del sistema di illuminazione:

- Metodo area: valuta congiuntamente gli ambienti (per edifici con 3 attività principali o attività che occupano più del $30 \%$ della superficie costruita).

- Metodo di attività: valuta individualmente ogni attività.

Poiché la Biblioteca Comunale ospita 3 attività principali e occupa oltre il $30 \%$ della superficie totale, abbiamo optato per il Metodo Area. La procedura di calcolo è avvenuta a partire dalle seguenti fasi:

a) Le principali attività sono state identificate secondo la Tabella del Limite Massimo Accettabile di Densità di Potenza di llluminazione (DPIL) per il livello di efficienza desiderato - Metodo dell'area dell'edificio, del Manuale per l'Applicazione di RTQ-C (2017). Per le attività non descritte, è stata scelta un'attività equivalente.

b) È stata individuata l'area di ciascuna attività, in metri quadrati.

RC: 100247

Disponibile in: https://www.nucleodoconhecimento.com.br/ingegneria-ambientaleit/biblioteca-comunale 
c) Quindi, è stata definita la potenza limite per ogni attività, moltiplicando l'area illuminata dal DPIL fornito nella stessa Tabella del Manuale, secondo l'Equazione 03. Dalla potenza limite per ogni attività, la somma dei poteri determinava la potenza limite dell'edificio.

d) Infine, la potenza totale installata nell'edificio è stata confrontata con la potenza limite per determinare il livello di efficienza dell'impianto di illuminazione utilizzando l'Equivalente Numerico previsto nella Tabella 06.

Tabella 06. Equivalente numerico (EqNum) per ogni livello di efficienza.

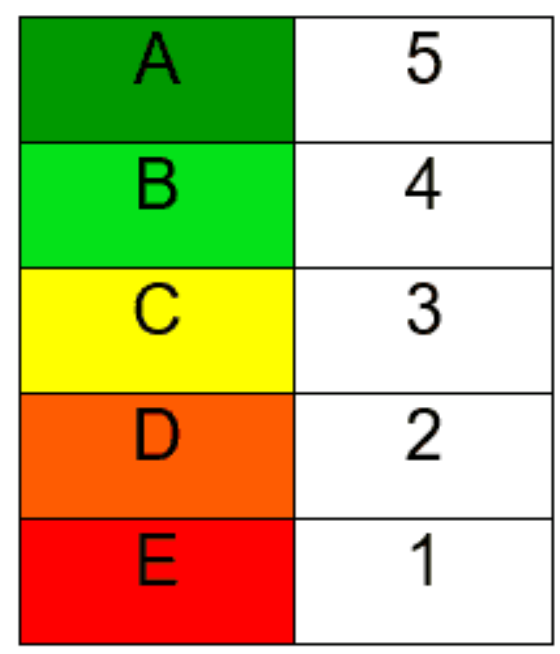

Fonte: Manuale dell'applicazione RTQ-C (2017).

\subsubsection{ARIA CONDIZIONATA}

Le apparecchiature di climatizzazione installate nella Biblioteca Comunale sono certificate da INMETRO, quindi sono state utilizzate le informazioni tecniche fornite dall'istituto stesso e dai produttori. La procedura per determinare l'efficienza del sistema è stata segregata in fasi:

RC: 100247

Disponibile in: https://www.nucleodoconhecimento.com.br/ingegneria-ambientaleit/biblioteca-comunale 
a) L'efficienza di ciascun sistema è stata osservata attraverso l'etichetta sull'apparecchiatura.

b) II coefficiente di ponderazione è stato determinato mediante il rapporto tra la potenza di ciascuna unità e la somma delle potenze di tutte le unità.

c) Dal coefficiente di ponderazione moltiplicato per l'equivalente numerico secondo la tabella 06, è stato ottenuto il risultato ponderato.

d) La somma dei risultati ponderati di ciascuna unità determinava l'efficienza totale, espressa nell'equazione 04.

Poiché ci sono efficienze distinte, il sistema di condizionamento dell'aria deve avere il suo equivalente numerico secondo la tabella 07 :

Tabella 07. Equivalente numerico per i sistemi di condizionamento e punteggio complessivo.

\begin{tabular}{|c|c|}
\hline$A$ & $>4,5$ a 5 \\
\hline$B$ & $>3,5 a<4,5$ \\
\hline$C$ & $>2,5 a<3,5$ \\
\hline$D$ & $>1,5 a<2,5$ \\
\hline$E$ & $<1,5$ \\
\hline
\end{tabular}

Fonte: Manuale dell'applicazione RTQ-C (2017).

RC: 100247

Disponibile in: https://www.nucleodoconhecimento.com.br/ingegneria-ambientaleit/biblioteca-comunale 


\subsubsection{PUNTEGGIO TOTALE}

La determinazione del livello di efficienza totale dell'edificio è espressa attraverso I'Equazione 05, che assegna pesi per ciascun impianto: 30\% per involucro, 30\% per impianto di illuminazione e $40 \%$ per impianto di climatizzazione. Dal risultato dell'equazione, è necessario convertire il valore numerico determinato per ciascun livello, come mostrato nella tabella 07.

\section{RISULTATI E DISCUSSIONE}

\subsection{INVOLUCRO}

Per analizzare l'involucro, è stata prima osservata la conformità con i prerequisiti specifici per questo sistema. La tabella 08 illustra il punteggio per ciascun prerequisito.

Tabella 08. Conformità ai prerequisiti specifici.

\begin{tabular}{|c|c|c|c|c|}
\hline \multirow{4}{*}{ Nível } & \multicolumn{2}{|c|}{ Transmitância Térmica (U) } & \multicolumn{2}{|c|}{$\begin{array}{c}\text { Cores e Absortância } \\
\text { Térmica }(\alpha)\end{array}$} \\
\hline & Cobertura & Parede & Cobertura & Parede \\
\hline & $\begin{array}{c}\text { Telha } \\
\text { fibrociment } \\
\text { o } 0.7 \mathrm{~cm} \mathrm{U} \\
=1.75 \mathrm{Wm}^{-} \\
{ }^{2} \mathrm{~K}\end{array}$ & $\begin{array}{c}\text { Tijolo } 6 \text { furos } \\
\text { circulares, } \\
\text { assentados na } \\
\text { maior dimensão. } U \\
=1.88 \mathrm{Wm}^{-2} \mathrm{~K}\end{array}$ & $\begin{array}{l}\text { Telha } \\
\text { fibrociment } \\
\text { o } 0.7 \mathrm{~cm} \alpha \\
=0.30\end{array}$ & $\begin{array}{c}\text { Vidro Incolor } \\
\alpha=0.25\end{array}$ \\
\hline & C & A & A & $A$ \\
\hline
\end{tabular}

Fonte: Paternità propria.

Successivamente, l'indicatore di consumo (IC) è stato determinato in base all'equazione 01 . La tabella 09 indica i valori per ogni variabile, ottenuti dal progetto architettonico.

RC: 100247

Disponibile in: https://www.nucleodoconhecimento.com.br/ingegneria-ambientaleit/biblioteca-comunale 
Tabella 09. Variabili di progetto.

\begin{tabular}{|l|c|}
\hline \multicolumn{1}{|c|}{ Variáveis de Projeto } & Valores \\
\hline Ape: Área de projeção do edifício $\left(\mathrm{m}^{2}\right) ;$ & $799.97 \mathrm{~m}^{2}$ \\
\hline Atot: Área total construída $\left(\mathrm{m}^{2}\right) ;$ & $1599.95 \mathrm{~m}^{2}$ \\
\hline Aenv: Área da envoltória $\left(\mathrm{m}^{2}\right) ;$ & $2641.75 \mathrm{~m}^{2}$ \\
\hline Apcob: Área de projeção da cobertura $\left(\mathrm{m}^{2}\right) ;$ & $1280.13 \mathrm{~m}^{2}$ \\
\hline AVS: Ângulo Vertical de Sombreamento & $0^{*}$ \\
\hline AHS: Ângulo Horizontal de Sombreamento; & $0^{*}$ \\
\hline FF: Fator de Forma, (Aenv/ Vtot); & 0.401 \\
\hline FA: Fator Altura, (Apcob/ Atot); & 0.194 \\
\hline FS: Fator Solar; & 0.27 \\
\hline $\begin{array}{l}\text { PAFT: Percentual de Abertura na Fachada total: média do } \\
\text { percentual de aberturas existentes; }\end{array}$ & $34.76 \%$ \\
\hline Vtot: Volume total da edificação (m3 ); & $6575.15 \mathrm{~m}^{3}$ \\
\hline
\end{tabular}

Fonte: Paternità propria.

* Valori nulli, perché l'edificio non presenta grondaie, né elementi verticali di protezione solare.

Per determinare il fattore solare, è stato utilizzato il vetro commerciale standard per edifici Cool Lite 114 PN 8 mm, come specificato nel Catalogo delle proprietà termiche e ottiche degli occhiali commercializzati in Brasile. Dopo il calcolo, è stato ottenuto I'Indicatore di consumo dell'involucro (IC) pari a 159.373.

Quindi, sono stati calcolati I'IC massimo ( $\left.I C_{\max }\right)$ e I'IC minimo (IC $\mathrm{C}_{\text {min }}$ ), utilizzando anche l'Equazione 01, ma sostituendo i valori descritti nella Tabella 04. Di conseguenza, si è concluso che $\mathrm{IC}_{\max }$ è uguale a 173.852 e IC min è uguale a 147.165.

Per calcolare I'Intervallo (i) IC max e IC min, è stata applicata l'Equazione 02, dove il risultato ottenuto è stato 6.671 .

RC: 100247

Disponibile in: https://www.nucleodoconhecimento.com.br/ingegneria-ambientaleit/biblioteca-comunale 
Dal risultato di (i), i valori di (i) della tabella 05 sono stati sostituiti da 6.671 , risultando nella tabella 10 , che presenta i limiti minimo e massimo per ciascun livello di efficienza.

Tabella 10. Valori ICenv massimi e minimi per ogni livello.

\begin{tabular}{|c|c|c|c|c|c|}
\hline \multirow{5}{*}{ Mínimo } & A & B & C & D & E \\
\hline & \multirow{4}{*}{$\mathrm{N} / \mathrm{A}$} & $\begin{array}{c}\text { IC máx }-3 i+ \\
0,01\end{array}$ & $\begin{array}{c}\text { IC máx }-2 i+ \\
0,01\end{array}$ & $\begin{array}{c}\text { IC máx - i + } \\
0,01\end{array}$ & IC máx $+0,01$ \\
\hline & & $\begin{array}{c}173,852-3 \\
(6,671)+ \\
0,01\end{array}$ & $\begin{array}{c}173,852-2 \\
(6,671)+ \\
0,01\end{array}$ & $\begin{array}{c}173,852- \\
6,671+0,01\end{array}$ & $173,852+0,01$ \\
\hline & & $\begin{array}{c}173,852- \\
20,013+ \\
0,01 \\
\end{array}$ & $\begin{array}{c}173,852- \\
13,342+ \\
0,01 \\
\end{array}$ & \multirow[b]{2}{*}{167,191} & \multirow[b]{2}{*}{173,862} \\
\hline & & *153,849 & 160,52 & & \\
\hline \multirow{5}{*}{ Máximo } & A & $B$ & C & D & E \\
\hline & IC máx - 3i & IC máx - 2i & IC máx - i & IC máx & \multirow{4}{*}{$\mathrm{N} / \mathrm{A}$} \\
\hline & $\begin{array}{c}173,852-3 \\
(6,671) \\
\end{array}$ & $\begin{array}{c}173,852-2 \\
(6,671) \\
\end{array}$ & $\begin{array}{c}173,852- \\
6,671 \\
\end{array}$ & \multirow[b]{3}{*}{173,852} & \\
\hline & $\begin{array}{c}173,852- \\
20,013\end{array}$ & $\begin{array}{c}173,852- \\
13,342 \\
\end{array}$ & \multirow[b]{2}{*}{167.181} & & \\
\hline & 153,839 & *160,51 & & & \\
\hline
\end{tabular}

Fonte: Paternità propria.

* Valore effettivo della Libreria IC env con i limiti trovati, dove il livello di efficienza dell'involucro è $B(160,51<159.373$ > 153.849).

\subsection{SISTEMA DI ILLUMINAZIONE}

Per determinare il livello di efficienza del Sistema di llluminazione utilizzando il Metodo area, sono state identificate le attività sviluppate in ogni spazio della Biblioteca. II rispetto dei prerequisiti è stato osservato singolarmente per ogni ambiente, infine, l'analisi del sistema nel suo complesso è stata eseguita attraverso la somma del DPIL di tutti gli ambienti. La procedura di calcolo si è basata sulle informazioni contenute nella tabella 11.

RC: 100247

Disponibile in: https://www.nucleodoconhecimento.com.br/ingegneria-ambientaleit/biblioteca-comunale 
Tabella 11. Classificazione del Sistema di Illuminazione.

\begin{tabular}{|c|c|c|c|c|c|}
\hline \multicolumn{2}{|l|}{ Atividade } & $\begin{array}{c}\text { Biblioteca } 1^{\circ} \\
\text { Pavimento }\end{array}$ & $\begin{array}{c}\text { Biblioteca } 2^{\circ} \\
\text { Pavimento }\end{array}$ & Escritório & $\begin{array}{c}\text { Total } \\
\text { Edifício* }\end{array}$ \\
\hline \multicolumn{2}{|l|}{ Área lluminada $\left(\mathrm{m}^{2}\right)$} & 559.6 & 282.48 & 359.63 & 1201.71 \\
\hline \multirow{4}{*}{ DPIL RTQ-C } & A & \multicolumn{2}{|c|}{12.7} & 9.7 & \multirow{4}{*}{$\mathrm{N} / \mathrm{A}$} \\
\hline & B & \multicolumn{2}{|c|}{14.6} & 11.2 & \\
\hline & C & \multicolumn{2}{|c|}{16.5} & 12.6 & \\
\hline & D & \multicolumn{2}{|c|}{18.4} & 14.1 & \\
\hline \multirow{2}{*}{ Lâmpadas } & $\begin{array}{l}40 \\
\text { W }\end{array}$ & 90 units & 104 units & 146 units & 340 \\
\hline & $\begin{array}{l}18 \\
W\end{array}$ & 142 units & 0 & 0 & 142 \\
\hline \multicolumn{2}{|c|}{ Potência Instalada (W) } & 6156 & 4160 & 5840 & 16156 \\
\hline \multirow{4}{*}{ Potência Limite (W) } & A & 7106.92 & 3587.49 & 3488.41 & 14182.82 \\
\hline & B & 8170.16 & 4124.2 & 4027.85 & 16322.21 \\
\hline & C & 9233.4 & 4660.92 & 4531.33 & 18425.65 \\
\hline & D & 10296.64 & 5197.63 & 5070.78 & 20565.05 \\
\hline \multicolumn{2}{|l|}{ Classificação Edifício } & \multicolumn{4}{|c|}{ Nível B } \\
\hline
\end{tabular}

Fonte: Paternità propria.

* L'Area Totale Illuminata dell'Edificio in metri quadrati rappresenta solo le aree di permanenza prolungata, cioè non include aree secondarie come, bagni, tettoia, circolazione e altri. Poiché le equazioni descritte nel Manuale per l'applicazione di RTQ-C prevedono già un margine considerando queste aree secondarie.

Secondo i calcoli DPIL di ciascun ambiente secondo l'equazione 03, il sistema di illuminazione è classificato come livello B. Tuttavia, considerando che l'edificio in studio non soddisfa i prerequisiti secondo il grafico 05 , il punteggio finale del sistema di illuminazione scende al livello C.

\subsection{ARIA CONDIZIONATA}

Per determinare l'efficienza del sistema di climatizzazione, i calcoli sono stati eseguiti secondo la metodologia e considerando che tutte le apparecchiature di

RC: 100247

Disponibile in: https://www.nucleodoconhecimento.com.br/ingegneria-ambientaleit/biblioteca-comunale 
condizionamento dell'aria della Biblioteca sono di tipo a soffitto diviso, sono stati ottenuti i seguenti risultati come mostrato nel grafico 12.

Tabella 12. Classificazione del sistema di climatizzazione.

\begin{tabular}{|c|c|c|c|c|c|c|}
\hline Ambiente & $\begin{array}{c}\text { Biblioteca 10 } \\
\text { Pavimento }\end{array}$ & $\begin{array}{c}\text { Biblioteca 2 } \\
\text { Pavimento }\end{array}$ & \multicolumn{3}{|c|}{ Escritório } \\
\hline Fabricante & ELGIN & ELGIN & GREE & RHEEM & KOMECO & AUSTIN \\
\hline Quantidade & 3 & 2 & 2 & 2 & 1 & 1 \\
\hline $\begin{array}{c}\text { Potência } \\
\text { (Btu/h) }\end{array}$ & 60000 & 60000 & 9000 & 20000 & 57000 & 60000 \\
\hline $\begin{array}{c}\text { Classificação } \\
\text { Energética }\end{array}$ & $\mathrm{C}$ & $\mathrm{C}$ & $\mathrm{A}$ & $\mathrm{D}$ & $\mathrm{D}$ & $\mathrm{D}$ \\
\hline $\begin{array}{c}\text { Equivalente } \\
\text { Numérico }\end{array}$ & 3 & 3 & 5 & 2 & 2 & 2 \\
\hline $\begin{array}{c}\text { Coeficiente } \\
\text { de }\end{array}$ & 0.22 & 0.22 & 0.03 & 0.07 & 0.21 & 0.22 \\
\hline $\begin{array}{c}\text { Ponderação } \\
\text { Resultado } \\
\text { Ponderado }\end{array}$ & 0.66 & 0.66 & 0.15 & 0.14 & 0.42 & 0.66 \\
\hline
\end{tabular}

Fonte: Paternità propria. Dall'applicazione dell'equazione 04, il risultato ottenuto è stato 2,69, che, secondo la tabella 07 , è equivalente al livello $C$ di efficienza.

\subsection{PUNTEGGIO TOTALE (PT)}

Per definire il livello complessivo di efficienza energetica dell'edificio, i risultati di ogni singolo sistema sono stati sintetizzati nell'equazione 05. La tabella 13 mostra i valori per ogni variabile utilizzata.

RC: 100247

Disponibile in: https://www.nucleodoconhecimento.com.br/ingegneria-ambientaleit/biblioteca-comunale 
Tabella 13. Variabili di equazione 05.

\begin{tabular}{|l|c|}
\hline EqNumEnv & 4 \\
\hline AC & $1201,71 \mathrm{~m}^{2}$ \\
\hline AU & $1592,73 \mathrm{~m}^{2}$ \\
\hline APT & $182,86 \mathrm{~m}^{2}$ \\
\hline ANC & $\mathrm{N} / \mathrm{A}^{*}$ \\
\hline EqNumV & $\mathrm{N} / \mathrm{A}^{*}$ \\
\hline EqNumDPI & 4 \\
\hline EqNumCA & 3 \\
\hline B & $\mathrm{N} / \mathrm{A}^{*}$ \\
\hline
\end{tabular}

Fonte: Paternità propria.

* Le variabili non si applicano agli ambienti condizionati.

Il risultato PT è stato di 3.111, rientrando nel livello C di efficienza energetica.

\section{CONSIDERAZIONI FINALI}

Si conclude che la Biblioteca Comunale di Campo Mourão non presenta risultati soddisfacenti di efficienza energetica. Per ottimizzarne l'efficienza, si suggerisce:

- Intervento nell'involucro con installazione di elementi di protezione solare, come grondaie orizzontali e verticali, brezze, cobogo, tra gli altri.

- In relazione al Sistema di Illuminazione, si consiglia di dividere i circuiti, in modo da consentire lo spegnimento delle lampade vicino alle finestre per l'utilizzo della luce naturale.

- Per quanto riguarda il sistema di climatizzazione, è importante sostituire l'apparecchiatura corrente che non ha efficienza A, poiché il $36 \%$ delle apparecchiature ha una classificazione D.

RC: 100247

Disponibile in: https://www.nucleodoconhecimento.com.br/ingegneria-ambientaleit/biblioteca-comunale 
- Oltre a queste misure, si raccomanda anche la sostituzione delle piastrelle in fibrocemento con piastrelle termoacustiche.

- Per rendere l'edificio più sostenibile, è consigliabile installare piastre fotovoltaiche per la generazione di energia solare, poiché l'investimento iniziale viene compensato a breve termine, fornendo risparmio, bassa manutenzione e funzionalità.

\section{RIEFERIMENTI}

ASSOCIAÇÃO BRASILEIRA DE NORMAS TÉCNICAS. NBR 15220-2: Desempenho térmico de edificações: Métodos de cálculo da transmitância térmica, da capacidade térmica, do atraso térmico e do fator solar de elementos e componentes de edificações. 2008.

CONSELHO DE ARQUIETURA E URBANISMO DO BRASIL (Brasil). Governo torna obrigatório que edifícios públicos federais tenham energia eficientes. Disponível em: https://www.caubr.gov.br/governo-torna-obrigatorio-que-edificiospublicos-federais-sejam-energeticamente-eficientes/\#comments. Acesso em: 28 ago. 2021.

CONFERÊNCIA DE ESTUdOS EM ENERGIA ELÉTRICA. PROGRAMA BRASILEIRO DE ETIQUETAGEM: Breve Panorama. Uberlândia, MG: Universidade Federal de Uberlândia, 2019. 5 p. Disponível em: https://www.lunasat.top/ceel/evento/artigos/\#2019. Acesso em: 28 ago. 2021.

CENTRO BRASILEIRO DE EFICIÊNCIA ENERGÉTICA EM EDIFICAÇÕES. Catálogo de Propriedades Térmicas e Óticas de Vidros Comercializados No Brasil. Brasil: Universidade Federal de Santa Catarina, 2015. Disponível em: https://cb3e.ufsc.br/sites/default/files/projetos/etiquetagem/catalogopropriedades-vidros-comercializados-brasil-13032015_v2.pdf. Acesso em: 28 jul. 2021.

RC: 100247

Disponibile in: https://www.nucleodoconhecimento.com.br/ingegneria-ambientaleit/biblioteca-comunale 
DOS SANTOS JUNIOR, J.E. Antiga Rodoviária de Campo Mourão-PR. 1969. Fotografia.

FILHO, Jairo Jerônimo De Campos. Mapa Localização Biblioteca. Campo Mourão: 2021. Mapa em escala gráfica. Software QGIS V. 3.2.

Programa Nacional De Conservação De Energia Elétrica. Manual para aplicação do RTQ-C. 4 ed. Brasil, 2017.

HARADA, Magali. Bioclimatismo: Blog de Arquitetura Bioclimática. Disponível em: http://bioclimatismo.com.br/bioclimatismo/zoneamento-bioclimatico-brasileiro/. Acesso em: 05 jun. 2021.

IBGE - INSTITUTO BRASILEIRO DE GEOGRAFIA E ESTATÍSTICA. Censo Brasileiro de 2020. Rio de Janeiro. 2021.

DEBATES INTERDISCIPLINARES VII. O Papel das Conferências Internacionais Sobre o Meio Ambiente Para o Desenvolvimento dos Regimes Internacionais Ambientais: De Estocolmo A Rio +20. Palhoça, Sc: Unisul, 2016. Disponível em: https://www.researchgate.net/profile/Jose-Baltazar-AndradeGuerra/publication/301626018_Debates_Interdisciplinares_VII/links/571e496f08aeac ed7889df5e/Debates-Interdisciplinares-VII.pdf\#page=168. Acesso em: 20 ago. 2021.

Inviato: Agosto, 2021.

Approvato: Ottobre 2021.

RC: 100247

Disponibile in: https://www.nucleodoconhecimento.com.br/ingegneria-ambientaleit/biblioteca-comunale 